\title{
REZEPTFREIE ARZNEIEN
}

\section{Überlassen Sie die Auswahl nicht den Patienten!}

- Sparen ist in der Arzneiversorgung angesagt. Eine Option sind OTC-Präparate. Über den Umgang damit und die Funktion des Grünen Rezepts informiert ein Leitfaden des Hausärzteverbandes.

Eine umfangreiche Gesundheitsreform steht bevor, ein Arzneisparpaket ist geschnürt, ein Milliardendefizit im Gesundheitsfonds steht zu befürchten: Wie wenig sich Voraussetzungen - und Einschränkungen - für Vertragsärzte in den vergangenen sechs Jahren geändert haben, zeigt der nach wie vor aktuelle Leitfaden des Deutschen Hausärzteverbandes zur „Ärztlich empfohlenen Selbstmedikation" aus dem Jahr 2004.

\section{Drohende Deprofessionalisierung der Arzneitherapie}

Der von Prof. Klaus-Dieter Kossow und Dr. Axel Dryden verfasste Leitfaden entstand unter dem Eindruck der Ausgrenzung rezeptfreier Arzneimittel aus dem Leistungskatalog der Kassen durch das Gesundheits-Modernisierungs-Gesetz (GMG). In der damals neuen Situation mahnten die Autoren zu einem Verhalten, das nach wie vor Gültigkeit hat: Die Wirksamkeit eines nicht verschreibungspflichtigen Arzneimittels hat sich durch neue gesetzliche Vorgaben nicht verändert.
Vor allem mahnt der Leitfaden dazu, die mit dem Ausschluss rezeptfreier Präparate drohende Deprofessionalisierung der Arzneimitteltherapie nicht zuzulassen. „Patienten, die ihren Arzt aufsuchen, sprechen dessen Kompetenz an." Stellen Ärzte die Auswahl eines rezeptfreien Arzneimittels in das Belieben des Patienten, wird dieser sich unter Umständen an den Apotheker wenden. Kossow und Dryden appellieren an ihre Kollegen, mit ihren Patienten eine Therapievereinbarung zu erzielen und zu erläutern. „Hausärztliche Medizin ist sprechende Medizin. Nutzen wir dieses Instrument der Zuwendung und Fürsorge."

Ein Handzettel oder eine mündliche Empfehlung würden nicht ausreichen. Das der GKV-Verordnung ähnliche Grüne Rezept erfülle „optisch und psychologisch“ die Erwartungen der Patienten viel besser, heißt es im Leitfaden. „Der informierte Patient wird auf der genauen Belieferung seines Rezepts bestehen " und eine Substitution durch den Apotheker nicht zulassen.

Bis zur weitgehenden Ausgrenzung rezeptfreier Präparate als Kassenleistung entfielen gut $25 \%$ der von Hausärzten verordneten Arzneimittel auf diese Gruppe. Dabei werden OTC-Präparate vor allem wegen ihres guten Nutzen-RisikoProfils von Ärzten geschätzt.

\section{Wertvoll, auch wenn die GKV nicht zahlt}

Der Leitfaden ermutigt ausdrücklich da$z u$, ungeachtet der gesetzlichen Vorschriften eine bisher für angemessen erachtete Arzneitherapie beizubehalten ein Rat, der auch heute Gültigkeit hat: „Macht der Arzt sich seinem Patienten gegenüber dadurch unglaubwürdig, dass er seine bisherige Therapie nicht mehr für sinnvoll hält oder eine Verschreibung verweigert, werden diese Patienten nicht mehr seine Praxis aufsuchen."

Die ärztlich gestützte Selbstmedikation durch das Grüne Rezept hat auch vor dem Hintergrund steigender Eigenbeteiligungen eine wichtige Funktion: Versicherte benötigen eine Verordnung als Nachweis beim Finanzamt. Empfänger von Hartz-IV-Leistungen werden künftig in Härtefällen vermehrt ein Grünes Rezept nachfragen, um gegenüber Behörden einen Mehrbedarf nachzuweisen.

Angesichts der neuen Sparrunde, die nun ansteht, gilt ein Ratschlag des Hausärzteverbandes unverändert: „Bleiben Sie Verbündeter Ihres Patienten. Lassen Sie sich nicht aus dieser Rolle verdrängen!“ EB -

\section{Kleine Checkliste für den Sonnenbrillenkauf}

\footnotetext{
- Wenn das schöne Wetter Ihre Patienten nun wieder vermehrt ins Freie lockt, sollten Sie darauf hinweisen, dass nicht nur die Haut, sondern auch die Augen UV-Schutz benötigen. Die UV-Belastung der Augen kann u. a. die Entstehung eines grauen Stars begünstigen. Für den Kauf von Sonnenbrillen gibt die Bundesanstalt für Arbeitsschutz und Arbeitsmedizin folgende Empfehlungen:

- CE-Zeichen obligatorisch (bestätigt gesetzlich vorgeschriebenen UV-Schutz)

- UV 400 oder UV 100 deuten auf einen ausreichenden Schutz hin.
}

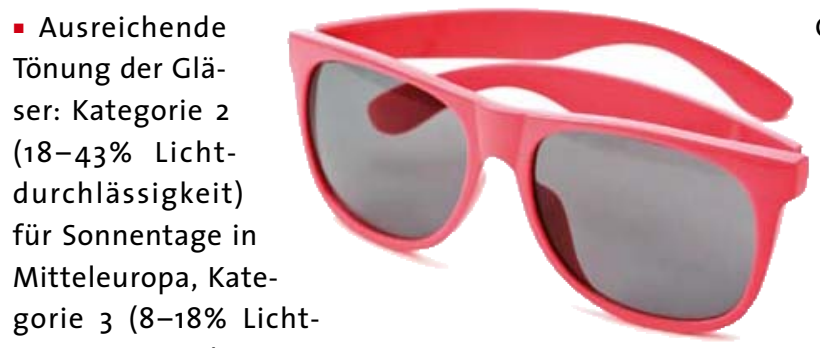

Gläser eignen sich zwar bei schlechten Sichtverhältnissen, sind aber oft für den Straßenverkehr ungeeignet.

- Die Gläser sollten die Augen gut abdecken, durchlässigkeit) für den Urlaub in den Subtropen und am Strand, Kategorie 4 für Skifahrer (3-18\% Lichtdurchlässigkeit, nicht für den Straßenverkehr geeignet!).

- Farbwahl: Empfehlenswert sind braune und graue Gläser. Gelbe, orange und rote d.h. mindestens bis zu den Augenbrauen und seitlich bis zum Gesichtsrand reichen. KE -

- Weitere Tipps unter www.baua.de/de/ Publikationen/Faltblaetter/Fg.html 